\title{
Naissance de la voix d'un tambour à fente chez les Mangbetu. Du geste de l'artisan à celui du musicien et du danseur
}

Birth of a Mangbetu slit drum. From the craftsman's hand to music and dance

Daniel Bariaux et Didier Demolin

\section{(2) OpenEdition}

\section{Journals}

Édition électronique

URL : http://journals.openedition.org/ethnomusicologie/1193

ISSN : 2235-7688

Éditeur

ADEM - Ateliers d'ethnomusicologie

Édition imprimée

Date de publication : 31 décembre 1995

Pagination : 105-114

ISBN : 2-8257-0537-3

ISSN : $1662-372 X$

\section{Référence électronique}

Daniel Bariaux et Didier Demolin, « Naissance de la voix d'un tambour à fente chez les Mangbetu. Du geste de l'artisan à celui du musicien et du danseur », Cahiers d'ethnomusicologie [En ligne], 8| 1995, mis en ligne le 04 janvier 2012, consulté le 02 mai 2019. URL : http://journals.openedition.org/ ethnomusicologie/1193

Ce document a été généré automatiquement le 2 mai 2019.

Tous droits réservés 


\title{
Naissance de la voix d'un tambour à fente chez les Mangbetu. Du geste de l'artisan à celui du musicien et du danseur
}

\author{
Birth of a Mangbetu slit drum. From the craftsman's hand to music and dance
}

Daniel Bariaux et Didier Demolin

1 Pour tout musicien, disposer d'un instrument de musique expressif constitue une préoccupation centrale. Cette quête se retrouve aux époques et dans les sociétés les plus variées, dans la facture de tous les instruments, des plus simples aux plus complexes. Beaucoup d'instrumentistes expriment cette quête sous une forme très simple: un instrument de musique doit être bon ${ }^{1}$.

2 Dans nos sociétés industrialisées, cette question de la bonne qualité des instruments de musique a préoccupé très tôt les esprits scientifiques, mais il faut bien constater que la réponse ne se trouve complètement ni dans les catégories mathématiques, physiques, physiologiques, psychologiques ou esthétiques, ni dans les expériences de laboratoire. Un mystère subsiste toujours : celui des gestes qu'accomplira le facteur d'instruments pour en créer un qui possède cette qualité.

3 Les gestes ont effectivement un caractère mystérieux. Pour le facteur, ils répondent à une logique stricte, spécifique et chargée de sens. Quand on observe des gestes, on peut bien sûr les décrire comme une succession de positions spatiales, mais pour saisir la cohérence de l'intention précise qui les guide, on doit nécessairement les accompagner extérieurement ou intérieurement.

4 Nous avons eu l'occasion de rencontrer et de suivre le travail d'un facteur de tambours à fente mangbetu (dans ce qui suit, nous l'appelerons par son nom : Mabondane) qui est un artisan réputé bien au-delà des frontières de son village. Sa démarche nous a paru exemplaire par sa cohérence et susceptible d'éclairer quelques points liés à la logique des gestes de la facture instrumentale en Afrique. L'observation du travail de cet artisan est 
d'autant plus intéressante qu'il vit dans une société de tradition orale où les rapports hommes-ancêtres-nature sont régis par une cosmogonie unifiante caractérisée, entre autres, par l'absence d'un métalangage abstrait pour parler des principes généraux qui régissent la facture et la musique. Cette absence d'un métalangage ne signifie pas pour autant l'absence de logique pour rendre compte de la subtilité et de la diversité d'un univers sonore.

Nous avons côtoyé Mabondane à une époque où il venait de recevoir la commande d'un nouvel instrument destiné à servir les besoins du chef, Danga Dambo, intronisé depuis peu. Au cours de nos rencontres, nous avons pu recueillir une série d'informations qui permettent de suivre l'ouvrage depuis le choix de l'arbre jusqu'au tambour fini. Nos connaissances actuelles ne nous permettent pas de décrire complètement la démarche de Mabondane dans les termes symboliques de sa propre cosmogonie. Nous avons donc opté pour une description dans laquelle il est possible de saisir la continuité du fil de ses pensées et de ses actes, en reprenant uniquement la succession d'images et de symboles qu'il utilisa lui-même pour caractériser les étapes cruciales de son travail.

6 Membre d'une société d'agriculteurs et de chasseurs, lui-même fils de chasseur, Mabondane se livre à deux activités principales : la chasse et la fabrication de tambours à fente d'un type appelé nekpokpo chez les Mangbetu².

7 L'instrument commandé devait être de grande taille afin de pouvoir envoyer des messages tambourinés depuis la cour jusqu'à des villages éloignés ${ }^{3}$. Il devait en outre s'intégrer à l'ensemble des instruments de musique de la cour de Danga Dambo.

Comme la plupart de ses proches, Mabondane est imprégné du savoir oral dont il a hérité. Ses dons de jeune joueur de tambour et sa curiosité l'on amené à s'intéresser à la fabrication de cet instrument et, très tôt attiré par la magie des gestes, il a observé l'adulte du village qui était facteur de tambour. Petit garçon, il a aussi régulièrement accompagné son père pour chasser et prospecter dans la forêt. Son exploration du grand territoire de chasse de la forêt équatoriale deviendra progressivement le théâtre mouvant d'expériences multiples. Sa confrontation aux végétaux, aux animaux, aux éléments lui donnera l'expérience nécessaire à l'épanouissement de son talent d'artisan. Sa vivacité et le contact quotidien avec des chasseurs expérimentés lui apprennent progressivement à lire dans le "grand livre de la nature »: apprentissage progressif et rigoureux dans sa cohérence car l'économie vitale du village dépend directement de la connaissance et du respect des lois qui gouvernent les relations du groupe avec la totalité de son environnement. Sa connaissance de l'univers de la forêt, de la nature en général et ses qualités humaines amèneront Mabondane à être intégré au groupe d'andoi, des ritualistes dont le pouvoir consiste à influencer favorablement le sort de ceux qui les contactent ${ }^{4}$.

Nous avons pu constater que la rigueur de sa démarche se prolonge aussi dans la sphère spirituelle. En effet, avant une chasse, Mabondane consulte l'oracle du mapingo ${ }^{5}$. Mais il lui pose d'abord une question dont il connaît avec certitude la réponse. Et ce n'est qu'après la réussite de ce test qu'il l'interroge sur des questions précises liées à l'organisation de la chasse future.

10 Cette lecture du grand livre de la nature possède un caractère d'autant plus exigeant qu'elle ne se pratique ni par lettres, ni par mots. Mabondane a appris à lire les geste de la nature. Les végétaux poussent et se métamorphosent au fil des saisons et des années. Un arbre croît, son fût se développe, ses fruits sont récoltés, un essaim d'abeilles se pose sur une de ses branches, la tempête le courbe, il gémit... Tous les gestes de cet arbre d'une 
espèce donnée vont permettre de le particulariser comme une individualité unique dont la multiplicité des traits de caractère formera progressivement un tempérament précis. Connaître cet arbre va consister à devenir capable d'entrer progressivement en contact avec cet être.

En l'abattant, Mabondane entame un nouveau mode de rapport avec lui. Dès les premiers chocs de la cognée, un corps à corps s'engage dans lequel, par ses actes, Mabondane va progressivement amener cet arbre du domaine de la nature à celui de la culture. Au travers du manche de la cognée, à chaque coup, il ressent les réactions de l'arbre et sonde ainsi les qualités du bois. Ses propres gestes commencent alors à l'informer sur l'être de l'arbre.

ondane vit dans une société où la fluidité des gestes n'a pas encore dû se soumettre aux contraintes rigides des lettres et des mots. Il n'a donc aucune difficulté à unir dans une connaissance de plus en plus globale l'information provenant de ses propres gestes à celles des gestes de l'être arbre.

Dès qu'il apprend la demande de fabrication d'un nouveau tambour, Mabondane se met à parcourir la forêt et à examiner les différentes essences (cordia millenii et pterocarpus soyauxii sont utilisés le plus souvent) susceptibles de lui donner le bois. De nombreuses fois, il a vu faire des instruments avec ces arbres ; lui-même en a déjà fabriqué plusieurs. C'est ainsi qu'il prendra deux mois pour « rencontrer» l'arbre dont il a besoin et pour annoncer au chef qu'il pourra exécuter la commande qui lui a été faite. Comme pour la chasse, c'est l'oracle du mapingo qui l'aide a délimiter la zone de la forêt où se fera sa recherche de l'arbre. Cinq spécimens sont repérés et marqués sur la base de leur apparence. Compte tenu de la grande taille du futur tambour du chef, l'arbre doit avoir un diamètre précis à mi-hauteur car ce n'est que dans cette portion du tronc que les fibres du bois conviennent; plus près des racines, le bois devient humide et les fibres grossières; plus près du houpier, le bois devient cassant et les fibres trop menues. Les nœuds dans le bois s'opposent à une propagation homogène des vibrations et il faudra inspecter l'écorce à la hauteur choisie pour bien vérifier qu'il n'y ait pas eu de branche latérale dans cette portion de tronc. Par contact, l'écorce épouse les fibres externes du tronc et sa structure donne une image de leur rectitude, de leur fermeté, de leur cohésion. Simplement en regardant les troncs, Mabondane peut « pré-voir » le tambour à l'intérieur du fût. Il va falloir poursuivre la sélection visuelle des spécimens en utilisant d'autres moyens d'investigation. Les arbres vont être auscultés. A l'aide du plat d'une cognée, du manche d'une herminette et d'un bâton lourd, Mabondane va systématiquement percuter les troncs à hauteur d'homme et écouter la réponse sonore de l'arbre. C'est par sa réponse résonante que l'arbre élu se manifeste au facteur. Voilà débuté un dialogue question-réponse entre Mabondane et le tambour qui ne se terminera qu'à la livraison de l'instrument. L'arbre sur pied vit, enraciné et tendu entre terre et ciel. La percussion de son fût l'ébranle et produit un son sourd. Son sourd, son latent, son potentiel, germe de la voix du tambour.

14 Mabondane a abattu l'arbre et va commencer à donner vie au tambour. Cette alchimie demandera une pleine et constante attention pour que le son-germe puisse se développer, s'extérioriser jusqu'à devenir la voix du chef.

15 L'arbre est couché sur le sol. Plusieurs aides assistent Mabondane dans son entreprise, mais l'artisan se comporte en maitre absolu dans toutes les étapes du déroulement de l'œuvre: il prend toutes les décisions, contrôle toutes les opérations et il est hors de question que quiconque exécute quoi que ce soit d'important en son absence. L'arbre à 
peine couché sur le sol, Mabondane parcourt déjà toute la longueur du tronc en le percutant et il délimite avec précision la portion médiane à extraire. Celle-ci est prélevée, écorcée et roulée jusqu'à une clairière voisine. On constate qu'il a déjà fixé la forme du tambour dans le bois car il fait arrêter la rotation et bloquer le rondin dans une position très précise qui correspond au tambour avec ses pattes en l'air. Le débitage de la partie supérieure commence en conservant de la matière pour les quatres pattes, grossièrement ébauchées. La pièce est retournée et mise sur pattes ; l'ébauche de la courbure des flancs et le creusement de la fente peuvent être entamés. Chaque coup de hache, chaque coup d'herminette permet d'éveiller à la vie sonore cet animal doté de quatre pattes, de deux flancs, d'une tête et d'une queue reliés par la fente-colonne vertébrale. Le bois a été travaillé dès l'abattage de l'arbre vert et il est jugé nécesssaire de laisser se reposer l'ouvrage après ce premier dégrossissage. Il faudra trois nouveaux mois pour qu'une lente transformation s'accomplisse. Les fibres gorgées de sève et d'eau qui maintenaient l'arbre debout par une tension intérieure vont lentement sécher et progressivement être tendues entre leurs nouvelles attaches : les parois latérales. Chaque percussion renseigne alors sur ce processus car, pour une forme donnée, la réponse de l'instrument « dit» le degré d'inversion des tensions dans le bois. Un végétal pousse du sol vers le ciel et possède toujours un sens de croissance précis; la fibre horizontale du bois couché conserve cette polarité établie entre la tête et la queue d'un animal, un crocodile ou un buffle, dont le sens virtuel de déplacement correspond à l'ancien sens de croissance de l'arbre. L'image d'un crocodile ou d'un buffle en mouvement devient le support d'une écoute dynamique des sons du tambour. Les gestes de l'animal, variés à l'infini, s'effectuent à partir d'un corps unique articulé autour d'un nombre fini de vertèbres et d'os divers. Comme un crocodile ou un buffle, le tambour va s'animer progressivement, grâce à de multiples percussions le long de son épine dorsale. Mabondane écoute le mouvement, la vie du son et conduit la sculpture jusqu'à une forme bien précise qui va permettre, sur cet instrument unique, la production de gestes sonores qui pourront aussi varier à l'infini. Le tambour est creusé, les flancs s'amincissent et plus l'instrument se vide de matière, plus il devient plein de son. Mais attention : la cage thoracique doit être proportionnée au reste du corps : un instrument trop lourd ne vibre pas assez et, s'il est trop léger, il ne porte plus le son. 
Fig. 1 : Les sons s'animent sous le battement des mailloches, le corps de l'instrument étant luimême animé par la dissymétrie des pattes et la tension de la queue.

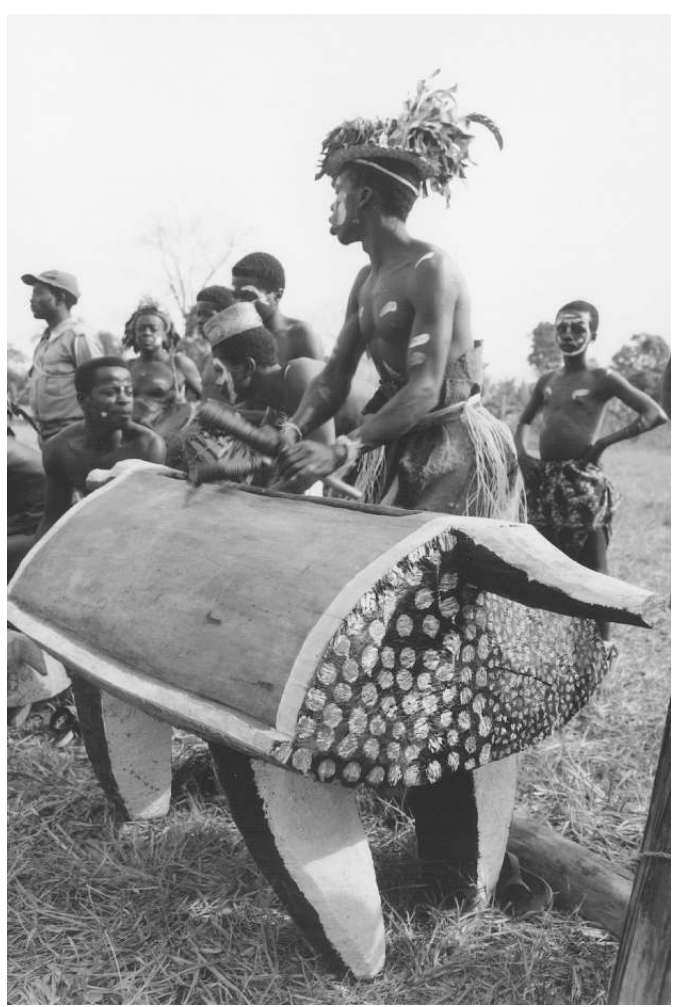

Photo : Didier Demolin

Le son germinatif du départ s'est développé. Le germe sonore "pré-entendu» par Mabondane dans l'arbre s'est transformé en gestes sonores animés. Une étape importante du travail se trouve accomplie : par sa maittrise, Mabondane est parvenu à unir sa vision dgeste végétal à sa perception d'un geste sonore.

Il lui reste une dernière étape à franchir, celle de donner une voix au tambour. Jeune enfant, il a appris à comprendre et à produire le langage tambouriné, et ces connaissances vont lui permettre de terminer son œuvre. Au départ, il a dû adopter un point de vue « végétal »; il a ensuite changé de niveau pour passer à l'« animal ». Il lui reste à pénétrer dans la dimension de l'articulation des sons parlés, c'est-à-dire la sphère des gestes humanisés. Commence alors une série d'ajustements de plus en plus fins, de plus en plus mystérieux dans leurs relations causales pour un observateur non averti : les pieds sont affinés, l'épaisseur du fond est réajustée à quelques endroits très précis, la tête est légèrement amenuisée, la fente est finement réalignée sur la direction des fibres, etc. Mabondane conduit lentement toutes ces opérations avec sûreté. Le sens de ses gestes de facteur tire sa cohérence de celui du discours qu'il parvient progressivement à articuler par son jeu. Plus question de frapper l'instrument avec le plat d'une cognée ; l'instrument est devenu un être et il ne parle que par le battement de mailloches. Ce tambour possède maintenant une voix individuelle; l'esprit qui l'anime permettra par son chant de soutenir la danse du chef et par ses paroles dson autorité.

La société occidentale ne connaît plus d'exemples où un facteur d'instrument a la possibilité de suivre toutes les phases de la métamorphose d'un produit naturel en un produit culturel. Les lois de la musique écrite nécessitent en outre toujours de lier les 
instruments les uns aux autres par des contraintes de diapason et de timbres. L'égalité du diapason et l'homogénéité des timbres contraignent les facteurs à tenir compte de mesures qui réduisent leur liberté dans la sphère de la créativité des gestes sonores.

La seule contrainte à laquelle Mabondane est soumis se situe sur un autre plan : son tambour doit posséder un esprit susceptible d'incarner la voix du chef. Peu importe la hauteur des tons mâle et femelle obtenus en percutant les deux flancs de l'instrument. Ici, il n'est pas question de mesures mais bien de proportions. La voix du tambour, la voix du chef ne se trouve pas dans les combinaisons plus ou moins complexes des tons hauts et bas. Elle se sert des alternances entre ces deux pôles sonores pour s'imprimer en creux dans l'espace où une infinité de gestes différents peuvent relier ces bornes toujours identiques.

Fig. 2 : Parmi les instruments de la cour, le nekpokpo se distingue non seulement par sa voix, mais aussi par sa taille.

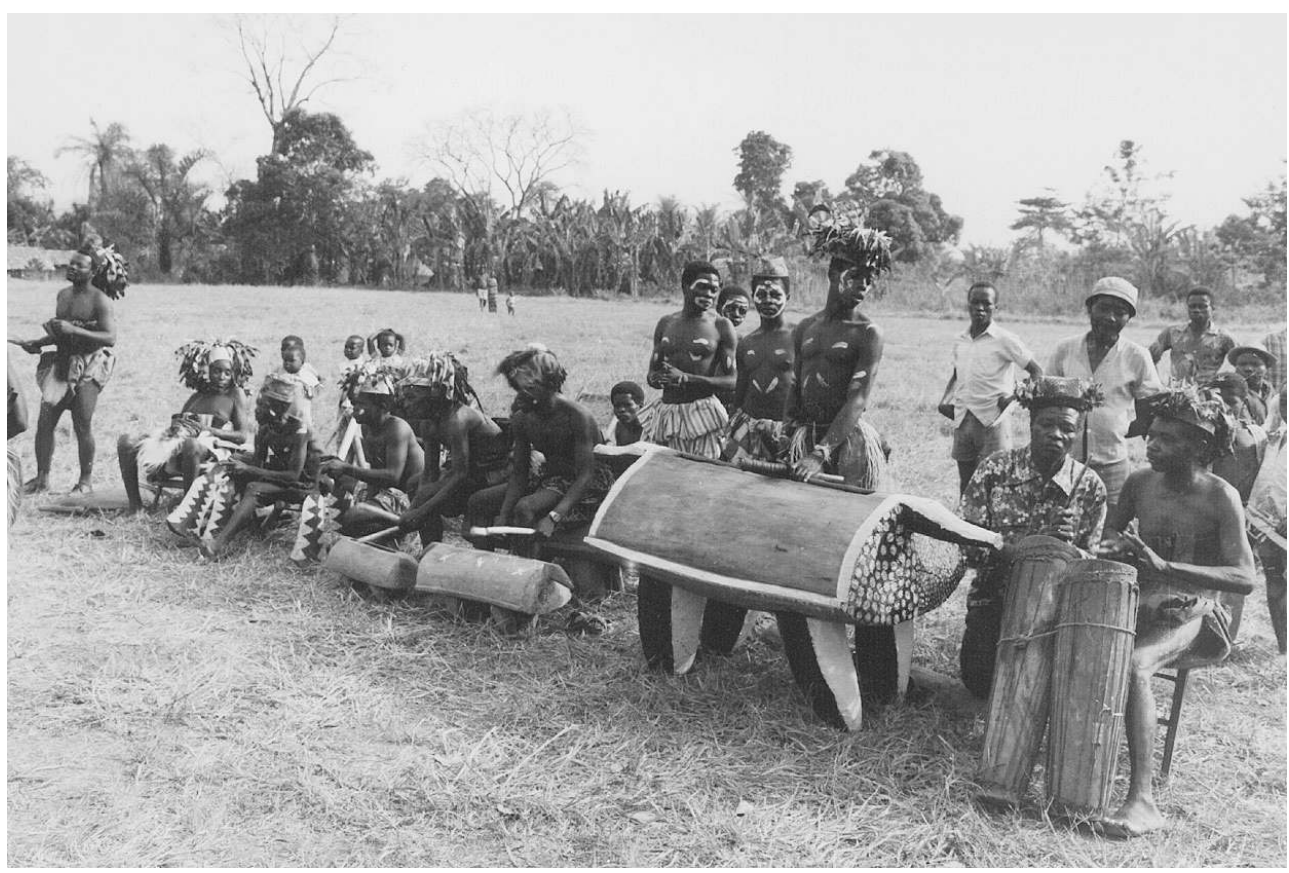

Photo : Didier Demolin

Les gestes de la parole articulée peuvent se traduire en gestes de percussion articulés; eux-mêmes se traduisent en gestes sonores tambourinés et articulés. L'oreille perçoit les gestes sonores de la voix et du tambour, le corps ressent les gestes de percussion, les yeux voient les gestes - les phases - de métamorphose des arbres et du développement de la forme du tambour.

Mabondane observe et étudie tous ces gestes. Il apprend à les accompagner par la pensée et, dans cet espace intérieur, il expérimente le dynamisme des gestes et leur fécondation mutuelle. Il apprend aussi à les accompagner par son propre corps qui danse, par sa main qui sculpte et à les traduire en formes extérieures dans l'espace extérieur. Mabondane organise les gestes, il les articule en un "corpus». Le fondement de sa démarche nécessite qu'il ressente en permanence ces gestes. Par ce savoir-faire, il peut intégrer consciemment ses propres gestes à ceux de tout son environnement. 
22 La voix de l'instrument stabilisée, Mabondane fera savoir au chef que l'instrument est prêt. Ses hommes ne viendront pas simplement prendre livraison du tambour, mais ils simuleront son enlèvement. La nouvelle voix du chef ne se reçoit pas, il faut la prendre. Les musiciens et les danseurs de cour, habillés en guerriers pour la circonstance, équipés de boucliers factices faits d'herbe tressée et de lances, arracheront l'instrument au facteur comme s'il s'agissait d'une conquête. Ils porteront le nouveau nekpokpo jusqu'à la résidence du chef, en le protégeant des attaques symboliques des spectateurs.

Le tambour de Mabondane va maintenant être intégré dans la pratique musicale de la cour de Danga Dambo. La voix du nekpokpo permet déjà d'incarner la sagesse du chef, mais elle va aussi l'aider à faire preuve de son habileté et de son intelligence grâce à la formation d'un nouveau couple : danse-rythme tambouriné .

Lors de la réception de l'instrument, Danga Dambo va exécuter un solo de danse dont les pas s'articulent sur un ostinato joué par tous les instruments à l'exception du nouveau nekpokpo. Le chef, sur la base de cet ostinato rigoureux, va conduire son corps dans une série complexe de figures coordonnées. Ces figures, langage de signes, montrent à tous les sujets présents sa dextérité et son intelligence. Intelligence liée à sa maîtrise des gestes et à sa capacité d'effectuer des figures toujours renouvelées ${ }^{7}$.

25 L'originalité de sa danse constitue un gage de l'originalité de sa personnalité. Il exprime l'autorité, et le tambour principal, le nouveau tambour du chef, seul parmi tous les instruments, va proclamer cette autorité. En effet, le musicien de cour qui le joue calque ses gestes instrumentaux sur les gestes dansés. La danse du chef commande le jeu du tambour; le son du tambour stimule la danse du chef. Le couple danse-rythme tambouriné s'unit: le tambour danse et le chef tambourine. Alors l'assemblée entière « voit et entend l'autorité » et la reconnaits ${ }^{8}$.

26 En fait, la voix mangbetu du tambour «contient» les gestes de l'artisan facteur, du musicien et du danseur. Et ses qualités, intrinsèquement bonnes, contribuent à la bonne harmonie de la communauté.

27 La richesse de l'univers de Mabondane et des Mangbetu, celui de la tradition orale, se révèle par le geste de l'artisan, qui échappe à la verbalisation. Depuis longtemps, cet univers intrigue les chercheurs issus d'un monde où tout se conçoit et se pense en termes d'analyse et de décomposition en unités significatives. Une des tâches les plus exhaltantes et les plus difficiles du travail de terrain est de découvrir, de mettre à jour et surtout de comprendre le monde de l'oralité, qui à ses principes et ses règles propres. La tâche du chercheur n'est pas seulement de matérialiser ou de transcrire ce qui est non-verbalisé, mais de décrire ce qui est un autre langage. Les unités de cet autre langage ne sont pas les mêmes que celles du monde de la verbalisation, mais il existe des principes généraux communs à des cultures très différentes. Ainsi lorsque Mabondane cherche le bois des arbres, il ne fait rien d'autre que le travail d'un luthier sculptant une table de violon en Europe. Leur démarche est identique et procède de la même logique. Le geste, qui apparait comme un constituant fondamental de l'oralité, se défini notamment par des caractéristiques spatio-temporelles qui font son originalité. C'est ce geste qui révèle la rigueur du travail de facture de Mabondane.

L'étude d'un instrument de musique peut être abordée sous des angles multiples. Dans le cas qui nous occupe, nous aurions pu, par exemple, étudier la répartition des tambours nekpokpo dans la société mangbetu, mesurer le plus grand nombre possible d'instruments, établir des typologies à partir de variations morphologiques, etc. Bref, récolter sur le 
territoire mangbetu, sur le terrain, les informations les plus précises possibles sur l'objet nekpokpo. Nous avons volontairement opté pour une démarche différente dans laquelle notre « terrain ", notre objet d'étude est devenu l'observation rigoureuse et sans à priori de la voie suivie par Mabondane pour construire un instrument. De fait, nous abordons l'objet « tambour à fente », trouvé sur le terrain, au travers du sujet Mabondane œuvrant sur ce terrain. Selon cette approche, nous choisissons de donner plus d'importance au comment qu'au pourquoi. Pour nous, un champ d'étude devient significatif à partir du moment où nous devenons capables de découvrir, au travers du vécu d'un individu lié à un terrain particulier, les éléments d'une démarche humaine à caractère universel. Nous n'avons donc pas hésité, à titre méthodologique, de confronter implicitement l'approche de Mabondane à celle d'autres facteurs d'instruments de musique, qu'il soient africains ou européens. Et cela dans la perspectiuve d'éclairer la question fondamentale à laquelle tout facteur se trouve soumis : comment former la matière pour la faire sonner?

Les limites de notre terrain se confondent ainsi avec les limites de nos connaissances et de nos expériences. Dans ce contexte, connaître consiste essentiellement à parcourir et à élargir notre propre terrain.

\section{BIBLIOGRAPHIE}

DESCHAMPS Roger, 1972, « Note préliminaire concernant l'identification anatomique des espèces de bois utilisées dans la fabrication des tambours à fente de l'Afrique Centrale ». Africa-Tervuren XVIII, $1: 15-18$.

DEMOLIN Didier, 1990, "The social organization Mangbetu music », in : African Reflections : Art of Northeastern Zaïre, E. Schildkrout et C. Keim (éds.), New Yorl : American Museum of Natural History ; Seattle, London : University of Washington Press : 195-208.

EVANS PRITCHARD Edward Evan, 1937, Witchcraft, oracles and magic among the Azande. Oxford : Clarendon Press.

SCHAEFFNER André, 1980, Origine des instruments de musique. Paris : Mouton.

STRAVINSKY Igor, 1935, Chroniques de ma vie. Paris : Denoël et Steele.

TIÉROU Alphonse, 1989, Dooplé, loi éternelle de la danse africaine. Paris : Maisonneuve et Larose.

\section{NOTES}

1. Au premier abord, l'adjectif «bon» peut sembler flou lorsqu'il s'agit de caractériser un instrument de musique ou le son qu'il produit. Nous le choisissons cependant volontairement car il se retrouve largement dans la plupart des traditions musicales. Son imprécision relative permet en contre-partie de qualifier avec rigueur la nature d'une démarche qui échappe à la verbalisation.

2. Le tambour à fente nekpokpo est constitué d'une caisse de résonance, d'une "tête ", d'une "queue », et de quatre "pattes » parfois remplacées par deux patins, le tout sculpté à partir 
d'une seule pièce de bois. Symboliquement, ce tambour à fente nekpokpo est associé, entre autres choses, à un crocodile, qui est un animal aussi dangereux dans l'eau qu'en dehors de l'eau En effet, le nekpokpo peut, lui aussi, être dangereux par les paroles qu'il diffuse dans la communauté. 3. Les informations que le chef transmet depuis sa résidence sont le plus souvent des appels pour des réunions d'anciens, des annonces de décès ou encore des appels pour la chasse ou pour le travail aux champs.

4. Ces andoi sont régulièrement conviés à chanter et à danser pour apporter un sort favorable lorsque de nouveaux objets, de nouvelles créations ou des événements importants se réalisent. Durant ces chants et ces danses, les andoi ingèrent l'écorce d'une plante appelée naando (achornea floribunda); cette ingestion se fait en mastiquant directement l'écorce ou en buvant une décoction. Cet excitant donne de l'énergie physique aux danseurs, mais amorce aussi un processus qui permet d'entrer en contact avec les forces sous-jacentes à l'univers végétal.

Le lien du monde végétal avec l'univers des ancêtres se manifeste dans le culte d'atolo consacré à ces ancêtres. Une des manifestations de ce culte est la mise en terre d'un arbre qui s'appelera atolo et qui deviendra le lien entre les vivants et les ancêtres. Les requêtes et le dialogue avec les ancêtres se font devant cet arbre. Le rite du naando est considéré comme une des manifestations de ce culte des ancêtres. Chez les Mangbetu, la première récolte d'un homme qui a quitté le foyer familial (ou son premier salaire) est offerte au père : il " paye son naando » et maintient ainsi le lien fondamental avec sa filiation.

5. L'oracle du mapingo est couramment utilisé par les Mangbetu pour connaître le sort futur de certaines activités ou pour trancher dans des questions de justice (cf. Evans-Pritchard 1937 pour des détails sur cet oracle).

6. Ceci n'est pas sans rappeler ce qu'André Schaeffner disait à propos du théâtre balinais, dans lequel se mêlent à la fois le parler, le chant et la danse, mais aussi les gestes des musiciens qui, en se tenant sur la scène, forment une part de l'action mimée. Pour Schaeffner $(1980: 14)$ la musique instrumentale suppose toujours la danse : « elle est danse »; " l'homme frappe le sol de ses pieds ou de ses mains, bat son corps en cadence, sinon l'agite en partie ou en entier afin de mouvoir les objets ou les ornements qu'il porte "; «la danse trouve là sa musique qui lui demeure intimement liée ». Pour Igor Stravinsky aussi (1935: 157), la vue du geste et du mouvement des différentes parties du corps qui produisent la musique sont une nécessité pour la saisir dans toute son ampleur.

7. «La danse africaine, c'est la répétition du geste appris, selon la tradition, c'est-à-dire non une simple imitation du maître, non une copie, mais une connaissance parfaite du geste, qui laisse le danseur libre à l'intérieur de la technique d'improviser et de répondre par des gestes admis à l'appel du cosmos selon son inspiration.

Le danseur traditionnel africain est en perpétuel dialogue avec le cosmos, et comme dans tout langage, il respecte les « mots » mais improvise, crée sa « phrase » [...] Telle est la véritable danse africaine traditionnelle, création et improvisation continuelles et toujours renouvelées à l'intérieur d'un cadre bien défini. » (Tiérou 1989 : 30-31).

8. Chez les Mangbetu, la symbiose du geste du danseur et de celui du musicien se manifeste pleinement dans toutes les danses, mais plus spécialement au cours des danses appelées nebopo et mabolo. Cette dernière a deux modes d'exécution: l'un populaire et l'autre de cour. Lorsque Schweinfurth fut reçu par le roi Mbunza en 1870, il eut droit à un extraordinaire solo de danse mabolo par Mbunza. Outre l'honneur qu'il lui faisait, le roi mangbetu lui montrait son habileté et son intelligence. En 1984, pendant l'intronisation du chef Danga Dambo, les autres chefs mangbetu invités exécutèrent, chacun à leur tour accompagnés par le même orchestre et le même tambourinaire, un solo de danse destiné à montrer leur intelligence et leur dextérité. Ce même rapport entre tambourinaire et danseur existe dans la danse de deuil nebopo qui est executée par les femmes. Appelées par les battements d'un tambour à peaux appelé nabita, les 
femmes viennent, chacune à son tour, exécuter, devant les musiciens, un solo de danse au cours duquel ce sont les gestes de leur corps qui commandent aussi le tambour.

\section{RÉSUMÉS}

Le tambour à fente joue un rôle central dans la société Mangbetu et, dès lors, sa facture nécessite une attention particulière. Nous décrivons les différentes étapes de la fabrication d'un tel instrument comme elles nous furent rapportées par Mabondane, un facteur renommé. Nous suivons pas à pas sa démarche rigoureuse pour donner une voix au tambour destiné au chef, à partir du choix de l'arbre dans la forêt jusqu'à la livraison de l'instrument terminé. Nous nous sommes particulièrement attachés à faire apparaître la logique de pensée qui permet à ce facteur de conduire concrètement son travail de bout en bout.

The slit drum plays a central role in Mangbetu society and its making thus requires special attention. We describe the different stages in the crafting of such an instrument as they were related to us by Mabondane, a drum maker of repute. We follow his strict methods, step by step, from the choice of a forest tree to the instrument's delivery to the chief for whom it had been intended. We attach particular importance to the logical thinking that enables the instrument maker to carry out his work from start to finish.

\section{AUTEURS}

\section{DANIEL BARIAUX}

Daniel Bariaux enseigne l'acoustique musicale à l'Université Libre de Bruxelles. L'objet principal de ses recherches concerne l'étude des instruments de musique. La particularité de sa démarche consiste à réaliser aussi bien des études par lesméthodes de l'acoustique que de pratiquer luimême la facture instrumentale. Ce double point de vue, confronté à celui desmusiciens, lui permet d'orienter ses travaux dans uneperspective méthodologique qui tient explicitement compte desniveaux de la communication musicale.

\section{DIDIER DEMOLIN}

Didier Demolin enseigne la phonétique, la phonologie et I'ethnomusicologie à l'Université Libre de Bruxelles. Il a effectué des recherches en Afrique centrale (Zaïre) et en Afrique de l'Est (Kenya). Ses recherches actuelles portent sur les langues et lesmusiques traditionnelles du nordest du Zaïre (Mangbetu et Pygmées Efe). Son intérêt porte, plus particulièrement, sur le rapport entre la mélodie des tons de la parole et celle du chant ;sur la description des cultures musicales, sur le timbre, ainsi que sur les principes mis en œuvre dans la fabrication des instruments de musique. 